\title{
Belphégor
}

Littérature populaire et culture médiatique

17 | 2019

Mutations des légitimités dans les productions culturelles contemporaines

\section{Une bande dessinée adulte? Usages et mésusages de la légitimation}

\section{Sylvain Lesage}

\section{(2) OpenEdition}

\section{Journals}

Electronic version

URL: https://journals.openedition.org/belphegor/1607

DOI: $10.4000 /$ belphegor.1607

ISSN: 1499-7185

Publisher

LPCM

Electronic reference

Sylvain Lesage, "Une bande dessinée adulte ? Usages et mésusages de la légitimation", Belphégor

[Online], 17 | 2019, Online since 19 April 2019, connection on 25 August 2021. URL: http://

journals.openedition.org/belphegor/1607 ; DOI: https://doi.org/10.4000/belphegor.1607

This text was automatically generated on 25 August 2021.

\section{(c)}

Belphégor est mis à disposition selon les termes de la Licence Creative Commons Attribution - Pas d'Utilisation Commerciale - Pas de Modification 4.0 International. 


\title{
Une bande dessinée adulte? Usages et mésusages de la légitimation
}

\author{
Sylvain Lesage
}

1 Au milieu des années 1970, la bande dessinée est, en France, en pleine recomposition. Après leur départ fracassant de Pilote, Nikita Mandryka, Claire Bretécher et Marcel Gotlib fondent en 1972 L'Écho des savanes, qui vise à les émanciper du carcan d'un illustré trop enserré dans les contraintes de la presse pour la jeunesse. En 1975, Fluide glacial et Métal hurlant prolongent cet élan d'émancipation d'une génération d'auteurs par rapport aux cadres des journaux enfantins ${ }^{1}$. Trois ans plus tard, le lancement d'( $\grave{A}$ Suivre) par les éditions Casterman témoigne de la récupération de cet élan initié au sein des avant-gardes par les plus installées des maisons d'édition ${ }^{2}$. Ce mouvement de refondation des contours et des publics de la bande dessinée est loin de se cantonner à la seule sphère de l'édition. C'est ainsi en 1974 qu'apparaît le Salon de la bande dessinée d'Angoulême, manifestation qui prendra au fil des années un rôle central dans l'institutionnalisation de la reconnaissance de la bande dessinée. La même année, celleci apparaît pour la première fois comme secteur distinct dans les enquêtes de branche $\mathrm{du}$ Syndicat national des éditeurs, marquant bien sa structuration comme segment éditorial, et son poids nouveau dans l'économie du livre (elle ne représente, en 1974, qu'1,6 \% du marché global de l'édition, hors cession de droits ; mais dès 1981, c'est plus de $3 \%$ du marché que pèse alors la bande dessinée ${ }^{3}$ ).

2 Si ce milieu des années 1970 correspond à un tournant dans l'histoire de la bande dessinée franco-belge, c'est également en raison de l'intérêt académique nouveau qu'elle suscite alors. En 1975, Luc Boltanski publie dans le premier numéro des Actes de la recherche en sciences sociales un article séminal consacré à « la constitution du champ de la bande dessinée ${ }^{4}$ ». Boltanski entend y étudier «certains des mécanismes qui accompagnent l'apparition d'un champ formé sur le modèle des champs de culture savante» et "observer les changements que la constitution [...] d'un champ relativement autonome [...] introduit dans les caractéristiques des producteurs, dans les propriétés formelles des œuvres et dans le type de relations que les producteurs entretiennent avec les œuvres ». En cela, la bande dessinée constitue l'un des 
archétypes de ces «arts moyens » qui permettent de bâtir la théorie de la légitimation, un jalon donc entre Un art moyen ${ }^{5}$ et Les Règles de l'art ${ }^{6}$.

3 Cet article a été abondamment discuté par la suite, en particulier par les sociologues qui ont pointé les limites des théories de la légitimation : Éric Maigret est notamment revenu en $1994^{7}$ puis en $2012^{8}$ sur cet article, ce qui dit bien, au-delà des polémiques qu'il a suscitées, son caractère fondateur'. Mon objectif ici n'est pas de discuter l'apport de ce texte à la sociologie de la culture, travail déjà largement mené, mais d'aborder la réappropriation de la théorie de la légitimation par les spécialistes de bande dessinée et les communautés de fans, en me concentrant sur un aspect plus particulier: l'apparition d'une «bande dessinée adulte». L'analyse que propose Boltanski de cette mutation procède, on le verra, d'un discours du Bildungsroman tel que l'entend Christopher Pizzino ${ }^{10}$, un récit qui postule une élévation progressive de médiums illégitimes vers la dignité de neuvième art.

\section{LUC BOLTANSKI ET L'ÉMERgENCE D'UNE BANDE DESSINÉE « ADULTE ॥}

4 La thèse centrale de Luc Boltanski tourne autour d'un basculement de la bande dessinée vers l'âge adulte. L'expression revient en effet à plusieurs reprises pour désigner à la fois le lectorat (p. 44), le style (le dessin obscène) et «la revendication d'un statut adulte » (p. 51). Le contexte, on l'a vu, explique largement ce parti-pris : l'époque est marquée par une indéniable effervescence créative, et une vitalité éditoriale remarquable. Ce «moment 1975 » s'inscrit d'ailleurs dans le prolongement d'initiatives plus anciennes. La relance d'Actuel en périodique contre-culturel (1970-1975) avait, assurément, ouvert la voie à L'Écho des savanes ou Métal hurlant. Lancé en 1964, Chouchou ambitionnait de s'adresser à la jeunesse structurée par la radio et la culture yéyé. En 1969, L'Hebdo Hara-Kiri traduit la recherche d'une expression graphique sortant des carcans de l'illustré enfantin, dialoguant davantage avec le dessin satirique, avec le calembour ou le texte politique. Le tournant des années 1970 est donc, plus largement, l'aboutissement d'une décennie de recompositions du paysage de la bande dessinée.

5 Cette effervescence, Boltanski la repère à trois séries de transformations: une transformation des espaces de production (l'apparition de revues orientées vers la transgression, le rôle joué par des éditeurs d'un type nouveau); un renouvellement générationnel des créateurs; enfin, une transformation du lectorat. Pour Boltanski, l'émergence d'une nouvelle génération de dessinateurs et de scénaristes est centrale dans la construction du champ :

Nés entre 1935 et 1940 et entrés sur le marché vers 1965 [ils] ont en commun d'importer sur le terrain de la BD une nouvelle forme de relation à leur activité [...]. Originaires pour la plupart des classes populaires ou de la frange inférieure des classes moyennes, scolarisés au moins dans l'enseignement secondaire (parfois dans l'enseignement supérieur) et/ou ayant acquis une culture scolaire en autodidactes, ils ont accédé aux échelons socialement inférieurs du système d'éducation artistique [...]; ils détournent sur la bande des ambitions censurées qu'une origine sociale plus élevée les inciterait à investir dans le champ de la peinture ou de la littérature. Socialement destinés à obtenir pour toute rétribution de leur compétence graphique l'accès à des activités sans autonomie ni prestige professions moyennes de techniciens du dessin, monteurs, lettreurs, dessinateurs industriels, coloristes etc. - ils trouvent dans la BD l'instrument d'expression symbolique le plus élevé dans l'ordre des légitimités culturelles auquel ils puissent 
raisonnablement aspirer et aussi un instrument raisonnable de mobilité sociale et d'affranchissement du salariat ${ }^{11}$. dans la bande dessinée comme fruit d'ambitions artistiques contrariées par l'extraction sociale modeste des dessinateurs ne résiste pas à l'analyse, comme l'a montrée l'analyse prosopographique très fine de Jessica Kohn, qui montre que, par exemple, les dessinateurs passés en Belgique par les instituts Saint-Luc de Liège et Bruxelles viennent plutôt de familles de professions libérales. Plus largement, expliquer que ces dessinateurs se rabattent sur la bande dessinée faute de mieux, "c'est ignorer qu'une partie non négligeable d'entre eux a en mains les cartes pour une carrière artistique traditionnelle ${ }^{12}$ ». Par ailleurs, l'idée d'une génération nouvelle est très largement à nuancer, ne serait-ce que parce qu'une partie des auteurs signalés par Boltanski luimême appartiennent à la génération précédente - comme Fred, né en 1931 et débutant dans le métier dès 1946, et que «les pratiques professionnelles continuent à se construire à partir de relations intergénérationnelles ${ }^{13} »$.

Il demeure cependant un deuxième versant à la lecture boltanskienne de la transformation du paysage de la bande dessinée dans les années 1970 : l'apparition d'un lectorat adulte, que Boltanski ne sépare pas de transformations stylistiques : «Le dessin obscène publié en-dehors des filières traditionnelles (Le Canard sauvage, L'Écho des Savanes, etc.), a pour propriété essentielle d'être interdit aux enfants. Il exprime la revendication d'un statut adulte, i.e. de dessinateur pour adultes, la recherche d'une novation esthétique réalisable par un simple déplacement des contenus ${ }^{14} »$. On le voit : l'émergence d'une bande dessinée «adulte» est, pour Boltanski, une affaire simultanément éditoriale, stylistique et une transformation du lectorat.

mutation du lectorat concerne, à en croire Boltanski, d'abord de grands adolescents ou de jeunes adultes qui disposent d'une réserve de temps libre, placés en position d'intermédiaires culturels. Ces "nouveaux adolescents" se trouvent, selon Boltanski, dotés par leur passage prolongé dans le système scolaire d'une «ébauche de disposition cultivée » les poussant à transférer ces dispositions acquises à l'école au domaine de la bande dessinée. Dès les années 1960, on trouve ainsi plusieurs traces de l'émergence indiscutable de nouvelles communautés de lecteurs, à commencer par le Club des bandes dessinées (1962) qui se constitue autour d'un double objectif: exhumer, rassembler, étudier voire rééditer les bandes dessinées de l'enfance des adhérents, celles de leur " Âge d'or »; mais aussi affirmer la dignité d'un art proclamé adulte. Ainsi, le club se fixe comme objectif de démontrer

que la bande dessinée est un art adulte, un moyen d'expression égal au cinéma ou à la littérature [...]. Des centaines de milliers de bandes dessinées sont parues depuis 1897 et [...] il est impossible que parmi elles il ne se trouve pas quelques œuvres d'art. Le rôle du Club est de les retrouver et de persuader les éditeurs jusqu'ici voués à l'enfance, qu'ils ont en face d'eux un énorme public adulte ${ }^{15}$.

Si les effectifs ne dépassent pas les quelques centaines d'adhérents, l'étude du profil de ces bédéphiles des premiers temps offre un aperçu précieux sur un lectorat adulte de la bande dessinée au début des années 1960. En 1964, le Club compte quatre cents adhérents, dont le doyen est né en 1897, et les plus jeunes sont nés en 1945 :

Plus de $78 \%$ des adhérents sont nés entre 1922 et 1939 inclus, plus de $55 \%$ entre 1926 et 1934. Le type parfait est né en 1928 (presque $10 \%$ des adhérents d'âge connu) et, à six ans, apprit à lire dans les premiers numéros du Journal de Mickey ${ }^{16}$. 
Cette bédéphilie naissante témoigne de la transformation des hiérarchies et des pratiques culturelles. En partie fondée sur une exhumation nostalgique, le mouvement témoigne de la poursuite assumée et militante de la lecture de ce que ses plus ardents défenseurs veulent nommer le "neuvième $\operatorname{art}^{17}$ ». Deux ans plus tard, le constat par plusieurs titres de presse d'un "phénomène Astérix ${ }^{18}$ " qui se serait abattu sur la France prend acte d'une banalisation de la consommation de bande dessinée par les adultes ${ }^{19}$. Publiées dans les pages de Pilote depuis 1959, les aventures d'Astérix et Obélix font également l'objet d'albums édités par Dargaud, qui battent alors tous les records en librairie; pour la seule année 1966, ce sont pas mois de 3,6 millions d'albums d'Astérix qui sont imprimés ${ }^{20}$ ! Le sens aigu de la promotion dont fait preuve Georges Dargaud, et la capacité de la série à s'adresser aussi bien à un lectorat enfantin qu'aux adultes, à travers des références empruntées à la culture scolaire et à l'actualité, font mouche. La télévision française, alors portée par une ambition forte de démocratisation culturelle, consacre plusieurs émissions au Gaulois moustachu. Intervenant dans l'émission d'actualité Panorama le 23 décembre 1966, René Goscinny et Albert Uderzo ne se privent pas d'ironiser : que reste-t-il de neuf à dire sur Astérix, après huit albums réalisés, 20 journaux s'emparant du thème, quatre chansons, un feuilleton radiophonique et 45 contrats publicitaires ${ }^{21}$ ?

11 En multipliant les parallèles entre la geste gaullienne et les aventures gauloises, L'Express en particulier met l'accent sur les tonalités adultes de la série, et les niveaux de lecture qu'elle offre aux lecteurs avertis : « les Français de 1966 ne se reconnaissentils pas, dans ces Gaulois irréductibles, courageux, teigneux, têtus, ripailleurs, bagarreurs et rigolards ${ }^{22}$ ? ». Le " phénomène Astérix » et l'audience considérable que les aventures de Goscinny et Uderzo rencontrent dans l'espace public et médiatique disent bien le changement de regard porté sur la bande dessinée : plus que d'autres, Astérix est sans doute la série qui se prête le mieux à constater de nouvelles pratiques de lecture, tant les observateurs sont immédiatement sensibles à la portée satirique de la série de Goscinny et Uderzo, et sa mise en scène de la société française des Trente glorieuses ${ }^{23}$.

De nombreux observateurs relèvent donc, entre le début des années 1960 et le milieu des années 1970, l'émergence d'un lectorat adulte de la bande dessinée, un processus qui irait, au choix, de Chouchou à Métal hurlant, du Club des bandes dessinées, bientôt rebaptisé Centre d'étude des littératures d'expression graphiques, au lancement d'(À Suivre). Ce constat d'une transformation des lectorats a depuis été repris et intégré à la vulgate de l'histoire de la bande dessinée, pour faire des années 1960 un moment charnière dans l'apparition d'une bande dessinée adulte. Patrick Gaumer, dans son Dictionnaire mondial de la bande dessinée, identifie ainsi ce basculement à la figure de Barbarella, l'héroïne de Jean-Claude Forest: «Barbarella est un symbole; elle est la première héroïne de ce qu'il est convenu d'appeler aujourd'hui la bande dessinée pour adultes $^{24}$ ». De même, Thierry Groensteen, dans La bande dessinée, son histoire, ses maitres identifie un moment où la bande dessinée accède à la dignité d'art pour adultes au point d'en faire un chapitre de son parcours historique ${ }^{25}$.

\section{DES PRATIQUES DE LECTURE ANCIENNES}

13 Le récit de l'apparition d'une «bande dessinée pour adultes » apparue au milieu des années 1960 tient cependant en bonne partie du mythe historique, pour deux raisons 
principales : d'abord, on ne dispose pas de sources fiables concernant le lectorat de la bande dessinée avant le début des années 1980. Il paraît donc bien présomptueux de postuler une mutation culturelle fondamentale alors même qu'on ignore quel était véritablement le lectorat de part et d'autre de cette charnière symbolique du milieu des années 1960. Au contraire, on dispose de sources, rares et déjà abondamment commentées, établissant la consommation de bandes dessinées par des adultes ; le récit autobiographique de Cavanna ${ }^{26}$, notamment, rappelle l'existence d'un lectorat adulte au début du XXe siècle. Le cas des parents de Cavanna, lecteurs de L'Épatant, a pu pousser à conclure un peu vite que ces pratiques étaient limitées aux milieux populaires. Une telle répartition de l'espace social de la lecture reprend cependant étroitement l'assignation à la minorité des pratiques populaires, et me parait à ce titre des plus suspectes. Dans l'attente de travaux plus approfondis sur l'entre-deux-guerres, on peut remarquer qu'il existe des pratiques de lecture adultes de productions culturelles a priori destinées à la jeunesse, dont on peut raisonnablement envisager qu'elles soient plus massives que les hiérarchies culturelles ne le laissent supposer. Bien entendu, cela n'empêche pas de constater qu'entre le milieu des années 1960 et le milieu des années 1970, un nombre croissant de publications s'adressent spécifiquement à ces adultes, marquant bien un tournant dans les espaces de publication.

Par ailleurs, si des interrogations demeurent quant aux pratiques de lecture, il ne fait aucun doute que des bandes dessinées destinées aux adultes existent bien avant les années 1960. On peut en repérer plusieurs formes principales; les feuilletons publiés dans la presse quotidienne nationale et régionale en sont sans la forme la plus massive quantitativement. Phénomène encore paradoxalement très méconnu, cette bande dessinée de presse de grande diffusion se destine en partie aux adultes : adaptations d'œuvres littéraires, récits de crimes et autres feuilletons sentimentaux constituent le lot commun de cette bande dessinée qui trouve sa place dans les pages de France-Soir, de L'Humanité, de La Voix du Nord, de Ouest-France et de dizaines d'autres titres ${ }^{27}$. En outre, bien qu'on ne dispose pas de chiffres précis sur le lectorat des publications de petit format, il paraît difficilement contestable que certaines d'entre elles soient destinées en partie au moins à des adultes, avant l'apparition des labels " pour adultes " qui fleurissent notamment sur les fascicules d'Elvifrance dans les années 1970. On peut rappeler enfin que Nous Deux est d'abord sous-titré, lors de sa sortie en France en 1947, "l'hebdomadaire du roman dessiné ", et introduit des «films en planches dessinées ", bandes dessinées au lavis aux mises en pages souvent audacieuses. Bien que le « roman dessiné » cède bientôt la place au très méconnu roman-photo, l'après-guerre a correspondu à une tentative originale pour faire exister une bande dessinée sentimentale reposant sur des codes iconographiques nouveaux, inspirés de l'esthétique hollywoodienne ${ }^{28}$. Des publications spécialisées strictement dans la bande dessinée s'adressent également à un public adulte, en particulier Héroïc-Albums, qui ambitionnent « de ne pas plaire seulement à l'adolescence ; ils veulent présenter des récits dessinés réalistes, aussi artistiques que possible, débarrassés de tout esprit ridiculement enfantin ${ }^{29} \%$. Les formes de bande dessinée pour adultes sont donc nombreuses dans l'après-guerre, et persistent largement dans les années 1950 et 1960 ; elles sont cependant peu connues, car elles appartiennent à la large galaxie de formes non adoubées par les bédéphiles et les historiens du $9^{\mathrm{e}}$ art. Les voies de la respectabilité - et donc de la patrimonialisation - passent par l'illustré et l'album, deux supports qui ont longtemps accueilli en priorité les récits en images pour les enfants. 
15 L'idée d'une bande dessinée " adulte », ou " pour adultes », telle qu'elle se manifeste à partir des années 1960, témoigne ainsi d'une ambition militante, celle d'en faire une forme culturelle distincte de la bande dessinée « tous publics ", c'est-à-dire enfantine, avec des thématiques et des esthétiques propres. La construction discursive d'une bande dessinée adulte résulte donc d'une tentative de mise à distance des contraintes et spécificités des industries culturelles. L'entreprise de légitimation du «neuvième art » s'accompagne ainsi de l'occultation stratégique des pratiques de lecture adultes de la bande dessinée avant les années 1960. Derrière ce vocable renvoyant à un âge adulte, c'est une catégorie socio-générationnelle qui est visée, celle des hommes éduqués, comme l'éclaire l'article de Luc Boltanski lorsqu'il évoque les recompositions du lectorat:

Sans perdre son public traditionnel - enfants, adultes des classes populaires et «parents » des classes supérieures, qui « empruntent » les bandes de leurs enfants et se les approprient sur le mode ludique et distant caractéristique de la relation des classes cultivées aux biens symboliques démunis de légitimité - la BD, un peu à la façon des films dits de " catégorie B ", westerns, policiers, " péplums ", etc., dans les années 50, tend à acquérir aujourd'hui un nouveau public doté de nouvelles dispositions propres à modifier le type de relation qu'il entretient avec elle ${ }^{30}$.

Dans l'analyse bourdieuso-boltanskienne de la structuration du champ, la présence d'adultes dans le lectorat de la bande dessinée dans les années 1950 ne constitue donc guère, au fond, qu'une modalité du vaste lectorat enfantin des récits en images. Le légitimisme qui pèse sur cette sociologie de la culture est ici singulièrement marqué, à rebours des intuitions de Richard Hoggart sur la capacité de tous, y compris les membres des classes populaires, à la distance critique, à l'ironie, à la consommation nonchalante ${ }^{31}$. La revendication d'un statut de «neuvième art » par les bédéphiles et sa validation par la sociologie bourdieusienne de la culture, se fait au prix de la construction d'une dichotomie entre bande dessinée " adulte » d'avant-garde d'un côté, et production de masse et anonyme de l'autre. L'existence d'une bande dessinée destinée spécifiquement à un lectorat adulte, susceptible d'appropriations diverses par des lecteurs aux compétences variables, n'est ainsi pas envisagée avant le milieu des années 1960 par Luc Boltanski, qui s'empare des chevaux de bataille des militants de la légitimation du neuvième art en même temps qu'il en décrypte les postures. Comme le relève Harry Morgan,

Pour ramener la BD d'avant 1968 à l'univers enfantin, il a fallu la réduire au strict domaine des illustrés pour la jeunesse qui éclosent à partir du début du XX⿳亠丷厂 ${ }^{e}$ siècle. Il faut entendre dès lors par " passage de la BD à l'âge adulte » non l'invention d'une bande dessinée plus évoluée [...] mais la constatation qu'une littérature enfantine (la seule admise comme BD) a pu proposer à un certain moment des œuvres lisibles par tous les âges [...] et que, parmi les grandes revues enfantines, il en est une [Pilote] qui s'est adressée petit à petit à un public plus âgé (de lycéens et d'étudiants plutôt que d'écoliers et de collégiens) ${ }^{32}$.

17 Cette lecture légitimante du devenir historique de la bande dessinée s'est aujourd'hui largement imposée comme la vulgate de l'histoire du $9^{e}$ art, avec l'idée d'une bande dessinée adulte de Töpffer à l'apparition d'illustrés destinés à l'enfance au tournant du XXe siècle, une bande dessinée enfantine pendant un gros demi-siècle, et le retour d'une bande dessinée adulte dans les années 1960. 


\section{LE DEVENIR ADULTE DE LA BANDE DESSINÉE, DOUBLE INVERSÉ DE LA LOI DE 1949 SUR LES PUBLICATIONS DESTINÉES À LA JEUNESSE}

18 Cette lecture du devenir médiatique de la bande dessinée est d'autant plus convaincante qu'elle repose sur des évolutions socio-culturelles de grande ampleur, et notamment l'émergence de la jeunesse comme nouvelle catégorie sociale ${ }^{33}$, ou les recompositions des pratiques culturelles et médiatiques dans la France des Trente Glorieuses, et les débats féroces qu'occasionnent les industries culturelles entre apocalittici et integrati $i^{34}$. Mais cette construction d'une trajectoire idéalisée des cultures médiatiques ne reprend pas seulement le combat bédéphile : elle constitue également un double inversé de la loi du 16 juillet 1949 sur les publications destinées à la jeunesse ${ }^{35}$.

19 Cette loi, qui procède d'une entreprise de moralisation de la jeunesse, repose sur le présupposé que la bande dessinée ne saurait s'adresser qu'aux enfants ${ }^{36}$. La Commission de surveillance et de contrôle, chargée de l'exécution de la loi, observe ainsi dans les consignes qu'elle transmet aux groupes de presse que sa vigilance se justifie par le fait que «beaucoup de jeunes lecteurs actuels de la presse enfantine ont subi pendant la dernière guerre des chocs nerveux qui ont entraîné une extrême sensibilisation affective. Il y a lieu de ménager ces enfants et de ne pas les prédisposer aux névroses, non plus qu'aux terreurs paniques ${ }^{37} »$. L'adoption de la loi se fait donc dans un climat de « paniques morales ${ }^{38}$ » qui rend la bande dessinée responsable de la hausse supposée de la criminalité juvénile après-guerre. La loi s'inscrit ainsi dans une vision de la bande dessinée qui reprend, à peu d'éléments près, les termes du débat autour du romanfeuilleton dans les années $1830^{39}$ ou de la moralisation de l'écrit obscène sous la III ${ }^{\mathrm{e}}$ République ${ }^{40}$. Georges Sadoul, qui avait dès les années 1930 entamé le combat contre les illustrés « américains ", écrivait :

La censure cinématographique a interdit les films de gangsters parce que ceux-ci augmentaient, dit-on, la criminalité infantile. Si cette mesure était salutaire au cinéma, quelle mesure s'imposerait donc pour des récits comme Au service de la loi [...] ? L'apprenti boucher qu'on rencontre dans la rue, le nez collé sur Hurrah !, ne sera-t-il pas demain le précoce criminel qui s'est servi de son couteau pour tuer avant de voler, ou l'auteur d'une tentative de kidnapping ${ }^{41}$ ?

20 Les groupes de pression, le législateur, les membres de la Commission de surveillance et de contrôle : le consensus est quasi-général pour faire de la bande dessinée une lecture d'enfants, méritant à ce titre une surveillance étroite. La censure de Barbarella de JeanClaude Forest est un bon révélateur de cette incapacité à penser des lectures adultes de la bande dessinée ${ }^{42}$.

21 Si Barbarella est surtout connu à travers l'album édité par Losfeld, puis le film de Roger Vadim, l'album n'est en fait que la deuxième version d'un récit préalablement publié dans les pages de $V$ Magazine, revue érotique fondée après la guerre et dirigée par Georges-Hilaire Gallet, responsable de la collection «Le Rayon fantastique ». Celui-ci connaît bien Jean-Claude Forest pour lui avoir commandé des couvertures du « Rayon fantastique » et des illustrations de romans légers publiés dans les pages de V Magazine.

C'est donc lui qui avait sollicité Jean-Claude Forest pour publier dans son magazine dès le milieu des années cinquante; dessinateur extrêmement polyvalent, Forest recourt d'ailleurs à un style beaucoup plus conventionnel dans les pages du magazine que pour 
les couvertures du "Rayon fantastique ", où les audaces graphiques sont encouragées. Dans $V$ Sélections puis $V$ Magazine, Forest illustre des récits d'aventures érotiques, le plus souvent en cadre exotique, et y apprend le langage des corps dénudés. Du 7 mars 1962 au 7 janvier 1964, la revue publie en feuilleton une série commandée à Forest: «Barbarella ». Le magazine, pourtant dans l'œil du collimateur de la Commission, ne s'attire pas de remarque particulière au cours de cette publication. En revanche, la publication par Éric Losfeld des aventures de l'intrépide héroïne en album suscite l'ire des commissaires.

Le 11 mars 1965, la question que soulève cet album est débattue lors d'une réunion de la Commission de surveillance et de contrôle. Le rapporteur de l'ouvrage est suffisamment troublé par son contenu pour proposer de faire circuler le titre lors d'une suspension de séance, afin que l'ensemble des commissaires ait un aperçu de l'ouvrage, à défaut d'avoir pu le lire. Or le débat qui s'engage à la reprise de la séance met en lumière deux attitudes possibles à l'égard de ce type de publications. Au cours du débat, sont en effet successivement évoqués les facteurs relatifs "à l'aspect matériel (papier épais, reliure de qualité, présence d'une jaquette), au tirage et au prix élevé du livre $^{43} »(54 \mathrm{~F})$, dont les commissaires s'accordent à constater qu'il relève "d'un genre inédit ». Dans un premier temps, les commissaires semblent disposés à voir dans les caractéristiques formelles singulières du titre la marque du beau livre, qui jouerait en faveur de la tolérance.

Mais le représentant des Francs et Franches camarades et celle des éditions Vaillant, Raoul Dubois et Madeleine Bellet, disent craindre " qu'en se montrant indulgent on ne facilite la prolifération de publications similaires et pensent que, dès la minute où se rencontrent suffisamment de passages licencieux, il n'y a qu'à requérir les rigueurs de la loi ». Les deux commissaires communistes vont même plus loin :

Par sa présentation en bande dessinée, Barbarella peut induire en erreur des adultes voulant acheter un album pour leurs enfants. Une prohibition de vente aux mineurs et d'affichage ne fera donc que contribuer à préserver la moralité des moins de 18 ans et décourager les imitateurs éventuels d'une initiative qui risquerait, autrement, de faire école ${ }^{44}$.

La Commission finit par se ranger aux arguments des deux éducateurs et vote ce qui devrait signifier l'arrêt de mort du titre, en adoptant une triple interdiction de vente aux mineurs, d'affichage et de publicité pour le titre. Ni le prix, ni le format n'atténuent suffisamment pour la Commission la charge subversive véhiculée par l'héroïne. Au-delà du seul cas de Barbarella, la Commission condamne ici le principe même d'une bande dessinée pour adultes, qui semble constituer une catégorie impensable pour les commissaires. Tout au long de la décennie, les commissaires se refusent à envisager cette possibilité, y compris lorsque, statistiques à l'appui, Georges Dargaud requiert auprès de la Commission de sortir du cadre d'application de l'article 2 de la loi de 1949 sur les publications destinées à la jeunesse, pour que Pilote soit considérée comme une revue pour adultes, simplement assujettie aux mesures prévues à l'article $14^{45}$.

Cette cécité aux lectures adultes commence à se fissurer dans les années 1970. Ainsi, la publication du deuxième volume d'Ulysse de Jacques Lob et Georges Pichard, en 1975, suscite une critique mitigée : tout en reconnaissant que «cet album s'adresse aux adultes ", les commissaires déplorent qu'il " ressemble trop, par sa présentation, aux publications destinées à la jeunesse ", et décident par conséquent de convoquer l'éditeur ${ }^{46}$. En octobre 1976, Roland Garel loue «la grande qualité technique et artistique des dessins » de Métal hurlant sans s'attirer la réprobation de ses collègues, 
qui examinent au cours d'un long débat la requête d'un abandon de l'interdiction de vente aux mineurs de la revue, décidée par arrêté du 21 juillet 1976. Le représentant des dessinateurs insiste sur l'évolution dans laquelle s'inscrit cette publication :

La bande dessinée est aujourd'hui un genre en plein évolution, et que l'on a tort de croire, par nature, destiné aux seuls enfants. Elle s'adresse, au contraire, de plus en plus, à tous les publics, sollicités par ailleurs sans ménagement par d'autres formes de média, comme la télévision, qui n'hésite pas à livrer, sans discrimination aucune, le spectacle de scènes de violence. La bande dessinée [...] devient actuellement un média comme un autre, pouvant traiter n'importe quel sujet. Or, par exemple, la Bible présentée de cette manière pourra n'être pas exempte de pornographie ; de même, l'Histoire de France en bandes dessinées, dont la publication vient d'être entreprise, devra relater les moments sanglants qui ont marqué notre passé ${ }^{47}$.

L'interdiction de vente aux mineurs de Métal hurlant est alors levée - à une courte majorité. Cette relative valorisation d'un magazine dédiée à la science-fiction et à l'exploration graphique témoigne du chemin parcouru ; mais la tolérance minimale dont commencent à bénéficier certaines des publications pour adultes est en trompel'œil: elle ne s'accompagne que d'un resserrement de la surveillance sur les cibles les plus dominées, en particulier les petits formats «pornographiques» et les revues de super-héros.

Ainsi, pour revendiquer le statut adulte de la bande dessinée et son accession à la dignité de neuvième art, les bédéphiles des années 1960 entreprennent de construire un modèle de lecture savante et distanciée de la bande dessinée, prenant le contrepoint de l'assignation à l'enfance telle qu'elle était conçue par les législateurs de 1949. La reprise par Boltanski de ce schéma dans son article de 1975 entérine ce récit qui voit émerger, à la faveur d'évolutions socio-culturelles, un lectorat adulte, là où l'évolution fondamentale est plus probablement l'apparition de lectures «cultivées" et non distanciées de la bande dessinée.

Cette construction discursive de l'émergence d'une bande dessinée adulte a trouvé d'efficaces relais qui en ont assis la solidité. Le marché de l'édition tend ainsi à se structurer à partir des années 1970 autour d'une polarité entre bande dessinée adulte novatrice d'un côté et industries culturelles de l'autre. Un éditeur comme Futuropolis, qui aimait à se rêver en "Gallimard de la bande dessinée », a été l'un des premiers à bâtir avec systématisme une dialectique entre création artistique et mainstream stéréotypé ${ }^{48}$. Mais ce que montre l'exemple de Futuropolis, c'est aussi une singularité française en matière de légitimation générationnelle de la bande dessinée. Car le cheminement d'une bande dessinée qui accéderait - discursivement, insistons-y - à l'âge adulte dans les années 1960-1970 se retrouverait sans peine ailleurs, à commencer par les États-Unis. Mais les trajectoires de légitimation sont en revanche fort différentes, tant les hiérarchies culturelles y sont construites différemment et tant les marchés y sont régis de manières distinctes.

Alors qu'aux États-Unis la revendication d'un statut adulte s'est opérée dans les espaces de l'alternative, de l'underground, cette construction s'opère aussi largement, en France, du côté des acteurs de l'édition ${ }^{49}$. Le lancement d'(À Suivre) en 1978 témoigne du rôle décisif des éditeurs dans ce processus. La revue, qui procède d'une tentative de redynamisation d'un catalogue vieillissant par l'éditeur tournaisien Casterman, offre un espace pour développer une bande dessinée plus "littéraire ", sur le modèle de $L a$ Ballade de la mer salée d'Hugo Pratt, publiée en feuilleton avant d'être rassemblée en album par Casterman en 1975. Dans son éditorial célèbre, le rédacteur en chef de la 
revue, Jean-Paul Mougin, affirme clairement le lectorat visé : "(À Suivre) n'est pas un "magazine pour adultes" avec le clin d'œil grivois qui s'attache à cette expression. (À Suivre) est simplement une revue adulte ${ }^{50}$ ». Éditeur majeur de la bande dessinée enfantine, de Tintin à Alix en passant par Les 4 As ou Petzi, Casterman opère ici un repositionnement spectaculaire, et dont on peut juger de la réussite au poids de l'éditeur tournaisien dans le palmarès du Festival d'Angoulême. Catalogue de prépublication plus que véritable entreprise de presse, (À Suivre) et les collections qui en sont tirées (« Les romans (À Suivre)» en particulier) constituent bien des machines à légitimer.

31 Aujourd'hui encore, la recherche sur la bande dessinée est largement prisonnière de cette dialectique du passage à l'âge adulte, qui se réapproprie, parfois sans l'interroger, une imagerie romantique de l'auteur ${ }^{51}$. La lecture boltanskienne de ce processus, empreinte de légitimisme culturel - la bande dessinée deviendrait "adulte" au moment où la bourgeoisie culturelle s'en empare, ses nouveaux auteurs ne seraient que des artistes frustrés qui rabattraient leurs ambitions sur un art « moyen »- entérine au fond le combat des bédéphiles davantage qu'il n'en déconstruit les présupposés. Cette revendication d'un art "adulte", qui repousserait donc dans les enfers culturels une bande dessinée "non-adulte », appartenant pleinement aux industries culturelles, se retrouve à différents moments de l'histoire de la bande dessinée.

L'étroitesse du canon des littératures graphiques faisant l'objet de recherches académiques constitue une conséquence directe de cette vision linéaire du devenir du " neuvième art ». Bart Beaty et Benjamin Woo ont montré la sur-représentation dans les comics studies d'une poignée d'auteurs identifiés à ce statut adulte de la bande dessinée, d'Art Spiegelman à Alison Bechdel en passant par Marjane Satrapi ${ }^{52}$. Si un tel examen n'a pas encore été mené de manière systématique de ce côté-ci de l'Atlantique, il y a fort à parier que les résultats n'en seraient guère différents (si ce n'est qu'on y trouverait des œuvres davantage perçues comme transgénérationnelles: Astérix et, bien sûr, Tintin). Il paraît donc essentiel d'amender notre histoire de la bande dessinée et, à cette fin, le corpus des œuvres sur lesquelles la recherche universitaire contribue à édifier pas à pas, qu'on le veuille ou non, le canon des littératures graphiques.

Christopher Pizzino l'observait à propos du cas américain : «l'histoire du médium n'est pas celle d'un développement naturel menant des premiers balbutiements du pulp à la maturité littéraire : c'est l'histoire d'un conflit dans lequel la bande dessinée a été en permanence lue par les adultes, mais en étant interdite, menacée de censure, exclue ou subordonnée à d'autres médias dans un contexte d'enseignement, et repoussée par tous les moyens aux marges de la culture ${ }^{53}$ ». Il montre, dans son étude, à quel point des œuvres étroitement identifiées au canon du graphic novel, en apparence blanchies sous le harnais de la légitimité, telles que Fun Home d'Alison Bechdel, sont porteuses des tensions héritées de l'assignation de la bande dessinée à la déviance, à une lecture pathogène. Une telle approche ouvre des perspectives très neuves à l'étude de la bande dessinée : le récit du passage à l'âge adulte - ce que Christopher Pizzino appelle «le discours du Bildungsroman »- a occulté des pans entiers du passé de la bande dessinée ; il voile également les tensions persistantes héritées des stigmates de 1949. 


\section{NOTES}

1. Jean-Baptiste Barbier, Métal hurlant - (À Suivre). La bande dessinée fait sa révolution, Landerneau, Fonds Hélène et Édouard Leclerc, 2013.

2. Sylvain Lesage, Gert Meesters (dir.), (À Suivre). Archives d'une revue culte, Tours, Presses universitaires François-Rabelais, « Iconotextes », 2018.

3. Voir sur ce point Sylvain Lesage, Publier la bande dessinée. Les éditeurs franco-belges et l'album, 1950-1990, Villeurbanne, Presses de l'ENSSIB, « Papiers », 2018.

4. Luc Boltanski, «La constitution du champ de la bande dessinée ", Actes de la recherche en sciences sociales, 1975, vol. 1, $\mathrm{n}^{\circ}$ 1, p. 37-59.

5. Pierre Bourdieu, Luc Boltanski et Robert Castel, Un art moyen : essai sur les usages sociaux de la photographie, Paris, Minuit, 1965.

6. Pierre Bourdieu, Les Règles de l'art: genèse et structure du champ littéraire, Paris, Éd. du Seuil, 1992.

7. Éric Maigret, «La reconnaissance en demi-teinte de la bande dessinée », Réseaux, 1994, vol. 12, $\mathrm{n}^{\circ}$ 67, p. 113-140.

8. Éric Maigret, "Bande dessinée et postlégitimité » dans La bande dessinée: une médiaculture, Paris, Armand Colin / INA, 2012, p. 130-148.

9. Voir également sur ce point Bart Beaty, Unpopular Culture. Transforming the European Comic Book in the 1990s, Toronto, University of Toronto Press, 2007.

10. Christopher Pizzino, Arresting Development: Comics at the Boundaries of Literature, Austin, University of Texas Press, 2016.

11. Luc Boltanski, « La constitution du champ de la bande dessinée », art. cit., p. 39.

12. Jessica Kohn, Travailler dans les petits Mickeys. Les dessinateurs-illustrateurs en France et en Belgique (1945-1968), Thèse de doctorat en histoire, Paris III, 2018, p. 117.

13. Ibid., p. 66.

14. Luc Boltanski, « La constitution du champ de la bande dessinée », art. cit., p. 51.

15. Francis Lacassin, compte-rendu de l'Assemblée générale du Club des bandes dessinées, GiffWiff $\mathrm{n}^{\circ} 7$, juillet 1963, p. 5. Sur la naissance de la bédéphilie en France, voir les travaux de Julie Demange, Les Pionniers de la bédéphilie française. Émergence et construction d'un mouvement bédéphile en France (1961- 1977), Thèse de doctorat en histoire sous la direction de Pascal Ory, Université Paris I, en cours.

16. Pierre Couperie, «Composition et comportement du club », Giff-Wiff n¹1, septembre 1964, p. 53.

17. Francis Lacassin, Pour un neuvième art, la bande dessinée, Paris, Union générale d'édition, «10/18», 1971.

18. SN, « Le phénomène Astérix », L'Express, 19 septembre 1966.

19. Outre L'Express, deux titres de presse majeurs consacrent des dossiers aux aventures du guerrier moustachu : Paris-Match qui accorde deux pages aux «triomphes d'Astérix » dans son $n^{\circ} 890$ du 30 avril 1966, et Candide, qui place Astérix en une de son $n^{\circ} 348$ de décembre 1966, sous le titre « la folie Astérix ».

20. Sylvain Lesage, « Astérix, phénomène éditorial : Du succès de librairie à la modernisation du marché de la bande dessinée en France ", dans Bertrand Richet (dir.), Le Tour du monde d'Astérix, Paris, Presses Sorbonne Nouvelle, 2011 http://books.openedition.org/psn/6993.

21. "Pano-rama-stérix », Panorama du 23 décembre 1966, https://www.ina.fr/video/ CAF86014409/pano-rama-sterix-video.html.

22. SN, « Le phénomène Astérix », art. cit. 
23. Une décennie plus tard, ces aspects sont développés notamment par André Stoll dans Astérix, l'épopée burlesque de la France, Bruxelles, Complexe, 1978.

24. Patrick Gaumer, « Barbarella », Dictionnaire mondial de la bande dessinée. Paris, Larousse, 2010.

25. Thierry Groensteen, "L'âge adulte », dans La Bande dessinée. Son histoire, ses maîtres, Paris / Angoulême, Skira Flammarion / Cité internationale de la bande dessinée et de l'image, pp. 101-127. Le chapitre couvre les décennies 1960-1970.

26. François Cavanna, Les Ritals, Paris, Belfond, 1978.

27. Alain Beyrand, De Lariflette à Janique Aimée: Catalogue encyclopédique des bandes horizontales françaises dans la presse adulte de 1946 à 1975, Tours, Pressibus, 1995.

28. Sylvette Giet, Nous deux : 1947-1997 : apprendre la langue du cour, Leuven / Paris, Peeters / Vrin, 1997. Voir aussi Jan Baetens, Pour le roman-photo : essai, Bruxelles, Les Impressions nouvelles, 2010, et Isabelle Antonutti, Cino del Duca: de "Tarzan » à «Nous deux», itinéraire d'un patron de presse, Rennes, Presses universitaires de Rennes, 2012.

29. Extrait des vœux de la rédaction pour l'année 1951, cité par Thierry Martens, Le journal de Spirou, 1938-1988 : cinquante ans d'histoire(s), Marcinelle, Dupuis, 1988, p. 59.

30. Luc Boltanski, « La constitution du champ de la bande dessinée », art. cit., p. 40.

31. Richard Hoggart, La Culture du pauvre. Études sur le style de vie des classes populaires en Angleterre, Paris, Minuit, « Le sens commun », 1970.

32. Harry Morgan, «Bande dessinée et enfance, histoire d'un préjugé » dans Harry Morgan, Principes des littératures dessinées, Angoulême, France, Éditions de l'an 2, 2003, p. 164.

33. Voir sur ce point notamment Ludivine Bantigny, Le plus bel âge? Jeunes et jeunesse en France de l'aube des « Trente Glorieuses » à la guerre d'Algérie, Paris, Fayard, 2007.

34. Umberto Eco, Apocalittici e integrati: comunicazioni di massa e teorie della cultura di massa, Milan, Bompiani (« Portico : critica e saggi »), 1964.

35. Voir aussi sur ce point Jean-Matthieu Méon, «L'illégitimité de la bande dessinée et son institutionnalisation : le rôle de la loi du 16 juillet 1949 ", Hermès, 2009, n 54, p. 45-50.

36. Thierry Crépin, Haro sur le gangster ! La moralisation de la presse enfantine, 1934-1954, Paris, CNRS, 2001.

37. « Recommandations de la Commission de contrôle », reprises dans « Annexes », Enfance, 1953, vol. 6, n ${ }^{\circ}$ 5, p. 495.

38. Le concept de paniques morales a d'abord été formalisé par Stanley Cohen, Folk Devils and Moral Panics, Londres, Routledge, 2005 [1972].

39. Lise Dumasy-Queffélec, La Querelle du roman-feuilleton: littérature, presse et politique, un débat précurseur (1836-1848), Grenoble, ELLUG, 1999.

40. Annie Stora-Lamarre, L'Enfer de la III République: censeurs et pornographes (1881-1914), Paris, Imago, 1989.

41. Georges Sadoul, Ce que lisent vos enfants : la presse enfantine en France, son histoire, son évolution, son influence, Paris, Bureau d'éditions, 1938, p. 39.

42. Je résume ici des éléments développés dans «Barbarella : révolution sexuelle ou révolution éditoriale?», dans Alexis Lévrier, Guillaume Pinson (dir.), Les Petits aventuriers du quotidien. Journalisme et bande dessinée, Bruxelles, Les Impressions nouvelles, à paraître.

43. Bernard Joubert, Dictionnaire des livres et journaux interdits par arrêtés ministériels de 1949 à nos jours, Paris, Éd. du Cercle de la librairie, 2007.

44. AN, 19900208, article 4, CSC, procès-verbal de la 68 ééance du 11 mars 1965.

45. AN, CSC, 19900208, article 6, PV de la 93e séance du 18 octobre 1972.

46. AN, CSC, 19900208, article 7, procès de la $106^{\mathrm{e}}$ séance du 22 octobre 1975

47. AN, CSC, 19900208, article 8, procès-verbal de la $110^{\mathrm{e}}$ séance du 13 octobre 1976.

48. Voir à ce sujet «Bravo pour l'aventure. Futuropolis : creuset de nouveaux formats, carrefour des indépendants », dans Sylvain Lesage, Publier la bande dessinée, op. cit., p. 271-303. 
49. Pour une analyse de la manière dont le vocable de graphic novel, dans les années 1970, se nourrit des tensions au sein du champ en cours de structuration de la bande dessinée aux ÉtatsUnis, voir Jean-Matthieu Méon, "Introduire le graphic novel, une ambition circonscrite : les premiers usages nord-américains de l'étiquette et leur péritexte ", Revue française d'études américaines, $\mathrm{n}^{\circ} 151,2017, \mathrm{p} .176-193$.

50. Jean-Paul Mougin, « Éditorial », (À Suivre) n¹, février 1978, p. 3.

51. Voir sur ce point Benoît Glaude, "Origines romantiques du statut culturel de la bande dessinée francophone ", dans Maaheen Ahmed, Stéphanie Delneste et Jean-Louis Tilleuil (dir.), Le Statut culturel de la bande dessinée. Ambiguïtés et évolutions, Louvain, Academia / L'Harmattan, 2017, p. 139-171.

52. Bart Beaty et Benjamin Woo, The Greatest Comic Book of All Time: Symbolic Capital and the Field of American Comic Books, New York, Palgrave Macmillan, 2016, p. 7.

53. Christopher Pizzino, Arresting Development, op. cit., p.3 (ma traduction).

\section{ABSTRACTS}

Cet article revisite la question de la légitimation telle que Luc Boltanski la mobilise pour analyser la constitution du champ de la bande dessinée dans le premier numéro des Actes de la recherche en sciences sociales. J'y montre la manière dont la sociologie bourdieusienne de la culture construit tout autant qu'elle décrit la question de la légitimité, en minorant les pratiques de lecture adultes et en adoptant une posture légitimiste. J'analyse également le pouvoir légitimant d'un article devenu totémique dans la recherche sur la bande dessinée, et les impensés de cette posture légitimante.

\section{INDEX}

Mots-clés: bande dessinée, légitimation, champ littéraire, culture de masse, censure

\section{AUTHOR}

SYLVAIN LESAGE

Univ. Lille, CNRS, UMR 8529 - IRHiS (Institut de Recherches Historiques du Septentrion) 